\title{
Time resolved measurements of the biased disk effect at an Electron Cyclotron Resonance Ion Source
}

\author{
K. E. Stiebing, O. Hohn, S. Runkel, L. Schmidt, and H. Schmidt-Böcking \\ Institut für Kernphysik der Johann Wolfgang Goethe-Universität, August-Euler-Straße 6, D-60486 Frankfurt am Main, Germany \\ V. Mironov and G. Shirkov \\ Joint Institute for Nuclear Research, PPL JINR, Dubna, Moscow Region, 141980, Russia
}

(Received 24 February 1999; published 10 December 1999)

\begin{abstract}
First results are reported from time resolved measurements of ion currents extracted from the Frankfurt $14 \mathrm{GHz}$ Electron Cyclotron Resonance Ion Source with pulsed biased-disk voltage. It was found that the ion currents react promptly to changes of the bias. From the experimental results it is concluded that the biased disk effect is mainly due to improvements of the extraction conditions for the source and/or an enhanced transport of ions into the extraction area. By pulsing the disk voltage, short current pulses of highly charged ions can be generated with amplitudes significantly higher than the currents obtained in continuous mode.
\end{abstract}

PACS numbers: $52.50 . \mathrm{Gj}, 29.25 . \mathrm{Ni}, 07.77 . \mathrm{Ka}$

\section{INTRODUCTION}

It has been shown that the performance of Electron Cyclotron Resonance Ion Sources (ECRIS) can reasonably be improved by using special techniques like gas mixing [1], a biased disk [2-5], covering the plasma chamber with $\mathrm{Al}_{2} \mathrm{O}_{3}$, or using a plasma chamber entirely made of aluminum [6]. All these methods serve to positively influence the source plasma or its conditions of extraction in such a way that increased currents of highly ionized atoms can be extracted. The biased disk is used especially often. It is mounted on axis inside the plasma chamber close to the plasma. The bias voltage is negative with respect to the plasma chamber. By varying disk position and voltage, conditions can be optimized to increase the extracted ion currents by factors of 2 to 20 [7].

At the Frankfurt $14 \mathrm{GHz}$ ECRIS, we have measured the time structure of the extracted ion currents as responding to short pulses of the biased-disk voltage. We observed that the ion currents respond to changes of the disk voltage within a few microseconds, which is much too fast to explain the increased ion currents in terms of enhanced ion breeding. We propose rather that the "biased disk effect," at least at the conditions given in the Frankfurt ECRIS, is due to the optimization of the ion extraction conditions and/or the better transport of ions into the extraction area. We assume that these improvements are due to the formation of a beam of (energetic) electrons that oscillate between the disk and the extraction electrode. For the case of argon/oxygen gas mixing, we also observed an overshoot of the ion current for high charge states at the moment when the bias was switched on. For $\mathrm{Ar}^{12+}$ ions, an enhancement of the current by a factor of 3 was observed for ion-pulse durations of typically $20 \mu \mathrm{s}$.

\section{EXPERIMENT}

\section{A. Experimental setup}

The Frankfurt $14 \mathrm{GHz}$ ECRIS is described in detail elsewhere [8]. The magnetic mirror ratio is 2.9 at the extraction and 3.8 at the microwave-injection side. The hexapole field amounts to $1.15 \mathrm{~T}$ at the plasma chamber wall. The plasma chamber diameter is $58 \mathrm{~mm}$, its length, defined by the hexapole, is $190 \mathrm{~mm}$. The extraction electrode aperture has a diameter of $8 \mathrm{~mm}$. For the measurements discussed here, the microwave power was chosen to be $400 \mathrm{~W}$ and the extraction voltage was $25 \mathrm{kV}$. With these parameters, the source produces up to $100 \mu \mathrm{A}$ of $\mathrm{Ar}^{8+}$ and $5 \mu \mathrm{A}$ of $\mathrm{Ar}^{12+}$ in dc mode.

Pure argon and argon/oxygen mixtures were used at operating gas pressures in the range of $0.8 \times 10^{-7} \mathrm{hPa}$ to $5.0 \times 10^{-7} \mathrm{hPa}$ (measured at the pumping chamber at the injection side). As expected for the "gas-mixing effect," the use of oxygen as supporting (mixing) gas resulted in strongly increased currents for high argon charge states (e.g., a factor of 5 for $\mathrm{Ar}^{12+}$ ). However, a very accurate adjustment of the partial gas pressures of the two components was required to obtain the optimal ion output.

The time structure of ion currents was measured after the analyzing magnet using a Faraday cup adjusted to $50 \Omega$ readout impedance. The cup currents were displayed on an oscilloscope using a $1 \mathrm{k} \Omega$ termination resistor. A digital camera recorded the oscilloscope display.

An iron plug of $40 \mathrm{~mm}$ diameter with a $10 \mathrm{~mm}$ o.d. $\mathrm{Al}$ core was installed in the chamber to be used as a biased disk and to simultaneously increase the mirror ratio of the magnetic field. It was water cooled and could be moved axially within $\pm 10.0 \mathrm{~mm}$. This disk could be biased up to $-800 \mathrm{~V}$ either in dc mode or periodically by steplike 
pulses with a repetition rate from $10 \mathrm{~Hz}$ to $1 \mathrm{kHz}$ and variable pulse widths in the range of 0.2 to $100 \mathrm{~ms}$. Rise (fall) times could be chosen to be 10 (100) $\mu \mathrm{s}$, or vice versa. The maximum output current of the pulse generator (10 mA) was well above the measured disk currents for $\mathrm{dc}$ mode for all cases discussed here. Both the pulse and the high voltage $(\mathrm{HV})$ generators were installed on the HV terminal of the source. The oscilloscope was synchronized with the pulse generator using an optical transmitter-receiver pair.

\section{B. Experimental results}

Prior to the measurements with the pulsed disk, experiments were performed with continuous disk voltages (dc mode) for both pure argon plasma and argon/oxygen $\left(\mathrm{Ar} / \mathrm{O}_{2}\right)$ gas mixing plasma. In Fig. 1, the biased-disk currents are displayed as a function of the biased-disk voltage for both cases. In the given circuitry, positive currents correspond to ions going to the disk and/or to electrons being emitted from the disk. In Fig. 2 the currents of extracted $\mathrm{Ar}^{8+}$ ions are given as a function of the biased-disk voltages again for both the above cases. For the sake of better resolution in the range of low biased-disk voltages, the full range of variation of the disk voltage is not shown in Figs. 1 and 2. Plasma conditions and ion optics had been optimized for the production of $\mathrm{Ar}^{8+}$. The gas pressure was $1.5 \times 10^{-7} \mathrm{hPa}$ for both cases. The ratio of partial pressures for $\mathrm{Ar} / \mathrm{O}_{2}$ mixing was approximately 1:1.

In $\mathrm{Ar} / \mathrm{O}_{2}$ mixing, the values of the disk currents generally are higher by a factor of 5 compared to the currents with pure argon plasmas, reaching values of more than $1.0 \mathrm{~mA}$. The highest currents of $\mathrm{Ar}^{8+}$ are obtained for bias voltages of approximately $-30 \mathrm{~V}$. Here, depending on the operation conditions, a local maximum of the biased-disk currents is observed at bias voltages in the range of -20 to $-30 \mathrm{~V}$, quite analogous to

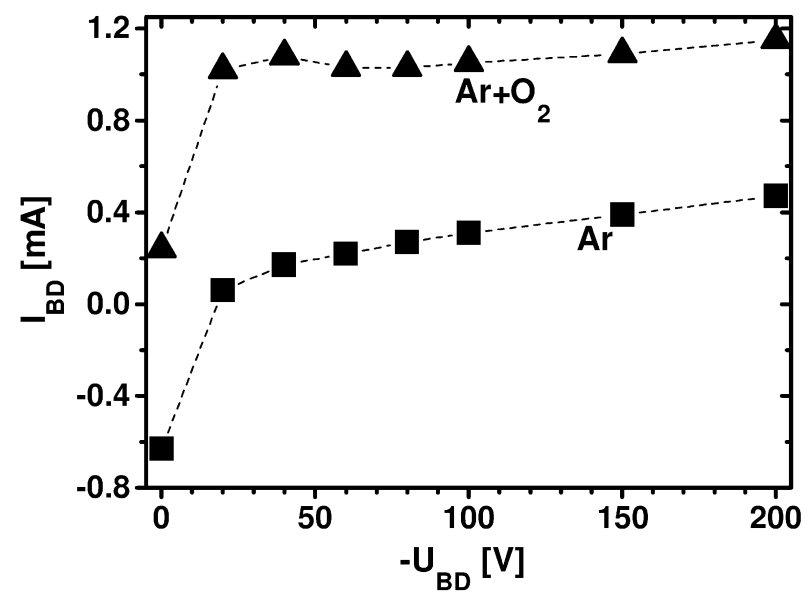

FIG. 1. Biased-disk currents as a function of the disk voltage for the case of plasmas of pure argon (squares) and $\mathrm{Ar}+\mathrm{O}_{2}$ gas mixing plasmas (triangles). results given in Ref. [9]. When the bias voltage exceeds the optimal value in this mode, strong oscillations of the extracted ion beam intensity can be observed with amplitudes in the range of $10 \%-50 \%$ of the absolute current magnitude. Oscillation frequencies were in the range of $10^{4}$ to $10^{5} \mathrm{~Hz}$. This instability definitely limited the ion currents. Optimal absolute bias values increase with the ion charge. They are close to zero for the range of $\mathrm{Ar}^{2+}$ to $\mathrm{Ar}^{6+}$ and reach $-60 \mathrm{~V}$ for $\mathrm{Ar}^{12+}$. Unlike the pure argon case, the relative increase of the extracted ion currents in gas mixing is only about a factor of 2, though the absolute values of currents exceed those for the pure argon working gas by a factor of about 5 for $\mathrm{Ar}^{12+}$.

For pure argon plasmas, optimal bias voltages are around $-200 \mathrm{~V}$ independent of the charge state. For high charge states, the extracted ion currents as a function of the biased-disk voltage increase by a factor of 8 compared to zero disk voltage. For voltages above the optimal value, the currents decrease slowly. In contrast to the gas mixing case, no oscillations of the beam current were observed in the above frequency range.

When the biased-disk voltage is applied in pulsed mode, the extracted ion currents, with some translational delay, saturate at the values measured for the case with the corresponding dc bias voltage. After bias voltage switch off, the currents drop to their respective levels of dc zero disk voltage. This process takes distinctly more time than given by the fast fall time of the pulser (see Sec. II A).

In Fig. 3, pulsed currents of $\mathrm{Ar}^{8+}$ ions extracted from pure argon plasmas are displayed for three different values of pulsed biased-disk voltages, taken with the rise- (fall-) time pattern $10(100) \mu \mathrm{s}$ of the pulse generator. At low biased-disk voltages, the rise time of ion currents is about $200 \mu \mathrm{s}$. This time decreases to values around $50 \mu \mathrm{s}$ for biased-disk voltages in the range of -200 to $-800 \mathrm{~V}$. Fall times are mainly due to the fall time of

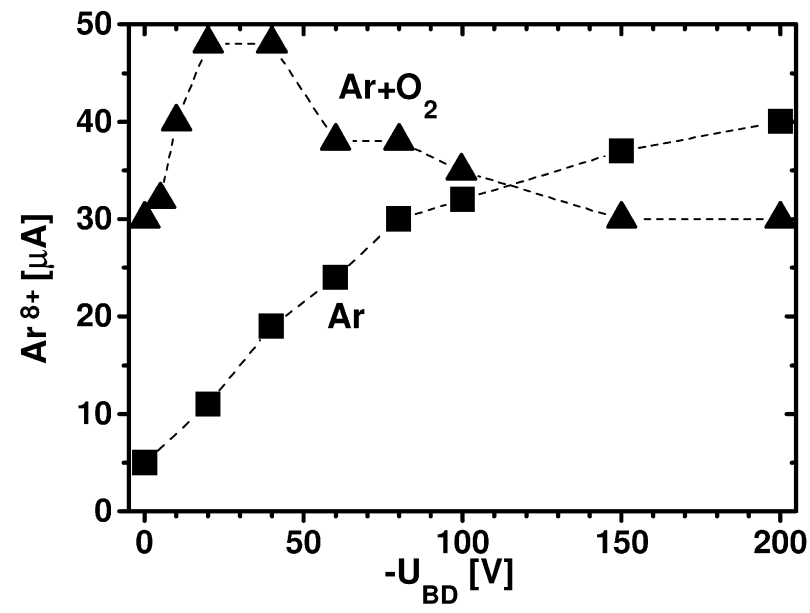

FIG. 2. Extracted currents of $\mathrm{Ar}^{8+}$ as a function of the disk voltage for the case of plasmas of pure argon (squares) and $\mathrm{Ar}+\mathrm{O}_{2}$ gas mixing (triangles). 


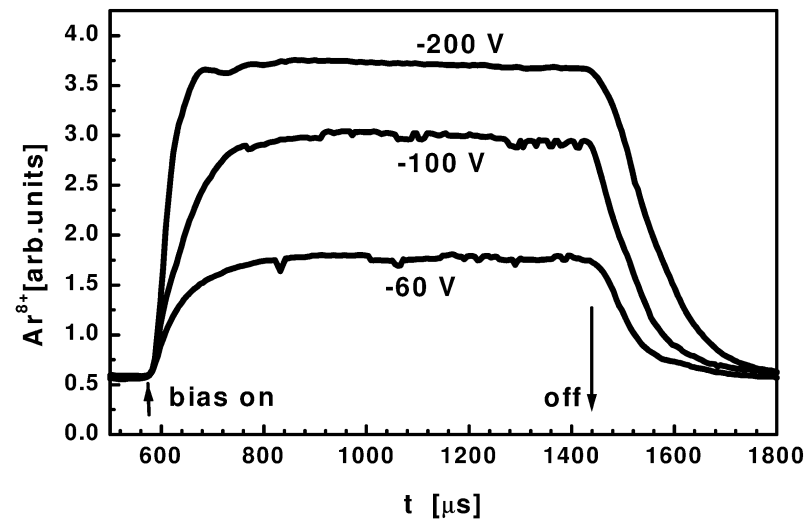

FIG. 3. Time structure of $\mathrm{Ar}^{8+}$ ion currents from pure argon plasmas for different amplitudes of the biased-disk voltage. The option with fast rise time $(10 \mu \mathrm{s})$ and slow fall time $(100 \mu \mathrm{s})$ of the pulse generator has been chosen.

the pulse generator at these conditions. Applying the reversed pattern of the pulse generator $(100 \mu \mathrm{s} / 10 \mu \mathrm{s})$, we have measured fall times close to $200 \mu$ s for all bias voltage amplitudes, indicating a nonlinear decay of the increased ion currents (Fig. 4). The shape of the ion-current pulses does not depend on the argon ion charge states. This is illustrated in Fig. 5 for the argon charge states $4+$ through $8+$ taken with the pulse pattern $10 \mu \mathrm{s} / 100 \mu \mathrm{s}$.

The situation changes drastically when $\mathrm{Ar} / \mathrm{O}_{2}$ gas mixing is applied. Here, the optimal dc bias voltages are different for different charge states in certain operational conditions. When the bias voltage is switched off, the currents of ions with lower charge states $\mathrm{Ar}^{\mathrm{Z}}$ (i.e., for $Z<8$ ) increase, whereas those for higher charge states $(Z \geq 8)$ decrease. When the bias is turned on, these processes reverse. This is illustrated in Fig. 6, where the

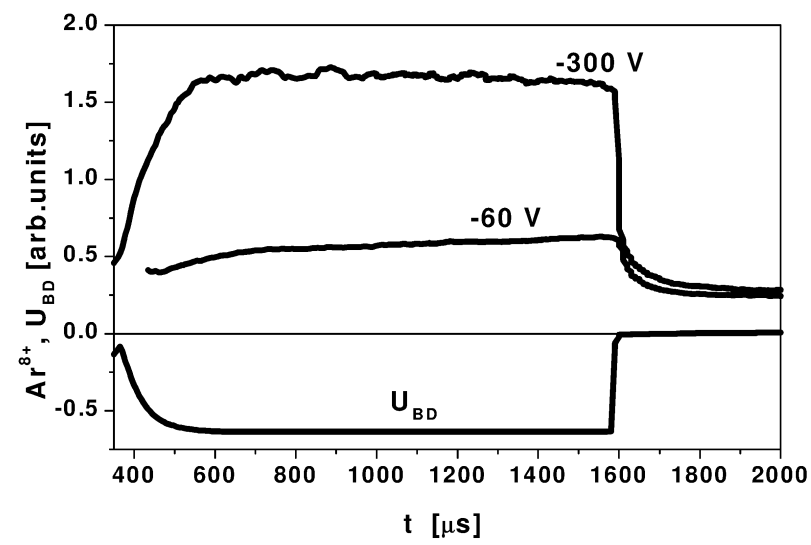

FIG. 4. Time structure of $\mathrm{Ar}^{8+}$ ion currents from pure argon plasmas for biased-disk voltages of -60 and $-300 \mathrm{~V}$. The bottom curve represents the shape of the biased-disk voltage pulse $U_{\mathrm{BD}}$. The option with slow rise time and fast fall time $(100 \mu \mathrm{s} / 10 \mu \mathrm{s})$ of the pulse generator has been chosen.

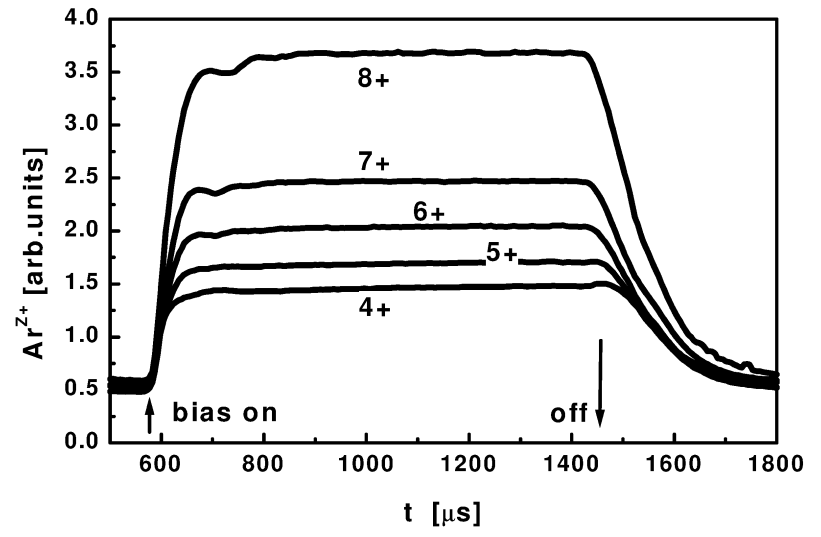

FIG. 5. Time structure of the extracted ion pulses for different charge sates from a pure argon plasma. The amplitude of the biased-disk voltage was $-300 \mathrm{~V}$.

current pulses for $\mathrm{Ar}^{4+}$ (representing low charge states) and $\mathrm{Ar}^{12+}$ (for high charge states) are compared.

Figure 7 shows, in better detail, how the $\mathrm{Ar}^{12+}$ ion current increases at the moment when the biased-disk voltage is switched on. (The de level of the current is indicated by the dashed line.) The amplitude of the biased-disk voltage pulse was $-200 \mathrm{~V}$. As already mentioned, the dc ion current showed oscillations at this biased-disk voltage. It was observed that this instability requires some time to develop (about $20 \mu \mathrm{s}$ ). By applying a biased-disk voltage distinctly larger than the value for the optimal dc ion currents, it is possible to get substantially larger amplitudes of extracted ion currents for a short period of time. The enhancement of the current amplitude for $\mathrm{Ar}^{12+}$ can be optimized to a factor of about 3 compared to the maximum values of the dc mode, mainly by carefully optimizing the $\mathrm{O}_{2}$ and Ar partial pressures. The rise times of about $10 \mu \mathrm{s}$, as measured so far, correspond to the rise time of the biased-disk voltage pulse.

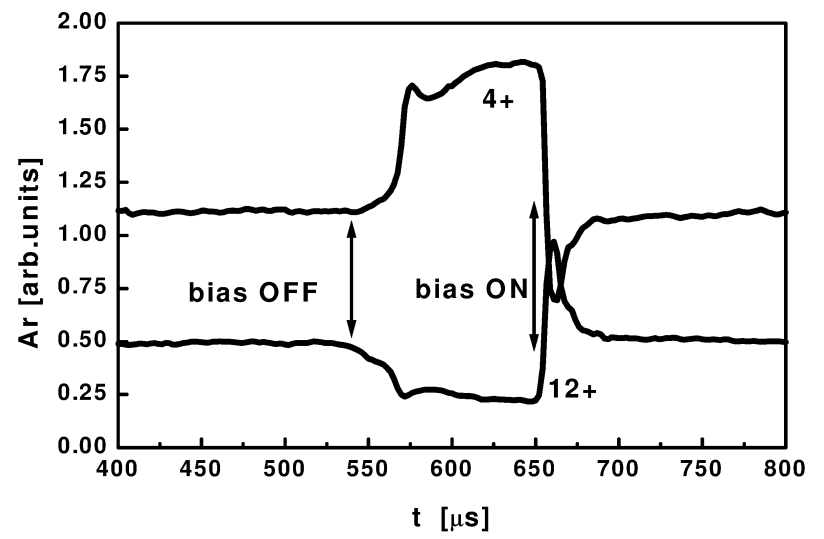

FIG. 6. Time structure of $\mathrm{Ar}^{4+}$ and $\mathrm{Ar}^{12+}$ ion currents from an $\mathrm{Ar} / \mathrm{O}_{2}$ (gas mixing) plasma. The amplitude of the biaseddisk voltage pulse was $-200 \mathrm{~V}$. 


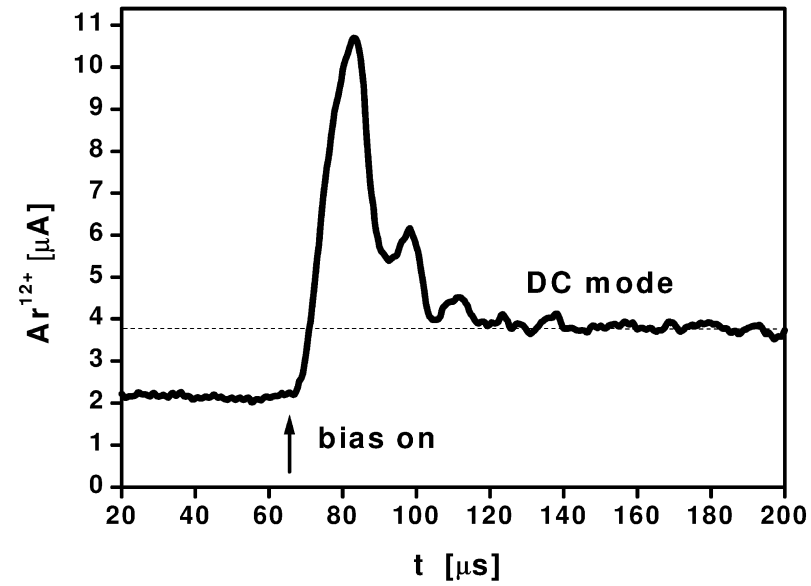

FIG. 7. Details of the time structure for a pulse of $\mathrm{Ar}^{12+}$ ions from an $\mathrm{Ar} / \mathrm{O}_{2}$ (gas mixing) plasma. The argon and oxygen partial pressures were optimized to produce the maximum pulse amplitude.

\section{CONCLUSION}

The extracted currents of highly charged ions respond remarkably fast to changes of the (pulsed) biased-disk voltage. Rise times as short as $10 \mu$ s have been observed, probably being entirely determined by the parameters of the biased-disk voltage pulse. For pure argon plasmas, the time structure of the measured current pulses is essentially independent of the ion charge states. Thus, one clearly has to conclude that the biased disk effect, at least at the conditions given here, is not connected to changes in the total ion density of the ECRIS plasma by additional ion breeding, as it was proposed earlier [4,5]. It rather has to be attributed to local changes of the ion density near the extraction area, which can be fast enough to account for the fast responses of the extracted currents.

In this article we do not attempt to fully explain the biased-disk operation. More detailed studies have to be conducted, are presently planned, and partially already started. As a first working hypothesis for future investigations, we would like to propose that the biased disk effect involves the creation of a beam of secondary electrons emitted from the disk and reflected by the electric fields at the extraction area and in front of the disk. These electrons are strongly bound to the magnetic field lines which, close to the axis, are dominated by the solenoidal field. Hence an oscillating electron beam along the axis of the ECRIS is built up when the biased-disk voltage is turned on. The space charge of such an electron beam depends on the number of reflections before the electrons leave the beam by scattering. It obviously reaches values that significantly change the parameters of the ionic transport in the plasma as well as the shape of the plasma meniscus near the extraction aperture of the ECRIS.

Changes of the extraction conditions under the injection of electron beams into an ECRIS have been observed earlier [10,11]. In Ref. [10] it is reported that the energy spread of oxygen ions in the extracted beams was substantially increased, while simultaneously the plasma potential decreased. Electron currents as high as $17 \mathrm{~mA}$ with $200 \mathrm{eV}$ electron energy have been used in these experiments. The energy spreads of the different ion components were proportional to their charge state and exceeded the typical values of ion temperatures in ECR plasmas by almost 1 order of magnitude. The authors explained this broadening of the energy spread by fluctuations of the plasma potential caused by the electron beam. It is interesting to note that gas mixing leads to similar changes in the values of the plasma potential and in the velocity distribution of extracted ions [10,12].

For the $\mathrm{Ar} / \mathrm{O}_{2}$ plasma, the increased biased-disk currents suggest that this plasma is denser in comparison to pure argon plasmas. At high biased-disk voltages, we observed ion-beam modulations. These oscillations may be attributable to electron-beam induced instabilities when the biased-disk voltage passes over a certain threshold value. This will restrict the available range of biased-disk voltages to lower values. For voltages above threshold, the excited oscillations of the secondary electron component reduce its density and hence the extracted ion currents. Therefore, the improvement factors are also restricted to values of only about 2 , as compared to a factor of 8 for the case of pure argon plasmas. Nevertheless, the very fast reaction of the extracted ion currents to changes of the biased-disk voltage allowed us to overcome this limit and to increase the pulsed currents for a certain period of time, shorter than the typical time of buildup of the plasma instabilities.

The presented pulsed-disk method is a very promising tool to investigate the mechanisms responsible for one of the most frequently used methods to increase the output of highly charged ions from ECR ion sources. More generally, the method of time resolved investigations of the beam formation from an ECRIS by pulsing the biased disk voltage is a way to obtain plasma parameters for ECR ion sources and to investigate plasma instabilities. As has been pointed out already, we plan a series of new experiments to provide further and complementary information on the mechanism and its influences on the creation and formation of beams of highly charged ions from our source (including emittance measurements). We also hope to get more conclusive information as to what extent the above model is correct.

From a practical point of view, the pulsed-disk method looks very promising to generate pulsed beams of highly charged ions with large current amplitudes, similar in result but different in mechanism and time scale to the well-known afterglow effect. It seems possible to produce enhanced quasi-dc currents, which, according to our experiment, could be modulated with frequencies of up to $1 \mathrm{MHz}$. It has to be found out to what extent this modulation can advantageously be used as a trigger for time resolved experiments or as "prebunching" for a 
subsequent pulsed accelerator structure. The method also seems suitable for ion beam stabilization.

\section{ACKNOWLEDGMENTS}

Valuable discussions with S. Biri, A. Drentje, and D. Meyer are gratefully acknowledged. The Frankfurt ECRIS-(ve)RFQ Facility is a HBFG project of Hessisches Ministerium für Wissenschaft und Kultur (HMWK) and Deutsche Forschungsgemeinschaft (DFG) Project No. III P 2-3772-116-246. This work was performed in the frame of a collaboration supported by wissenschaftlichtechnische Zusammenarbeit (WTZ), Bundesministerium für Bildung und Forschung (BMBF), Grant No. RUS669-97.

[1] A. G. Drentje, in Proceedings of the 11th International Workshop on Electron Cyclotron Resonance Ion Sources, Groningen, 1993, edited by A.G. Drentje (KVI Report No. 996), p. 155, and references therein.

[2] G. Melin et al., Proceedings of the 10th International Workshop on ECR Ion Sources, Knoxville, 1990, edited by F. Meyer (ORNL Report No. ORNL CONF-9011136), p. 1.

[3] S. Gammino, J. Sijbring, and A. G. Drentje, Rev. Sci. Instrum. 63, 2872 (1992).
[4] T. Nakagawa and T. Kageyama, Jpn. J. Appl. Phys. 30, L1588 (1991).

[5] K. Matsumoto, Rev. Sci. Instrum. 65, 1116 (1994).

[6] C. M. Lyneis, in Proceedings of the International Conference on ECR Ion Sources and Their Applications, East Lansing, Michigan, 1987 (NSCL Report No. MSUCP-47), p. 42; R. Geller et al. ibid., p. 1; T. Nakagawa, Jpn. J. Appl. Phys. 30, 930 (1991).

[7] Z. Q. Xie and C. M. Lyneis, Rev. Sci. Instrum. 65, 2947 (1994).

[8] K. E. Stiebing, H. Streitz, L. Schmidt, A. Schremmer, K. Bethge, H. Schmidt-Böcking, A. Schempp, U. Bessler, and P. Beller, in Proceedings of the 12th International Workshop on ECR Ion Sources, Tokyo, 1995, edited by M. Sekiguchi and T. Nakagawa (INS Report No. INS-J182), p. 122.

[9] D. P. May, Rev. Sci. Instrum. 69(2), 688 (1998).

[10] Z. Q. Xie and C. M. Lyneis, in Proceedings of the 11th International Workshop on Electron Cyclotron Resonance Ion Sources, Groningen, 1993, edited by A. G. Drentje (KVI Report No. 996), p. 106.

[11] S. Runkel, O. Hohn, L. Schmidt, K.E. Stiebing, H. Schmidt-Böcking, A. Schempp, and R. Becker, Rev. Sci. Instrum. 69(2), 721 (1998).

[12] A. Nadzeyka, D. Meyer, and K. Wiesemann, in Proceedings of the 13th International Workshop on ECR Ion Sources, College Station, Texas, 1997, edited by Donald P. May and Judith E. Ramirez (Texas A\&M University, College Station, TX, 1997), p. 185. 